\title{
Exact matched-beam envelopes for undepressed transport in a quadrupole lattice with unequal drift lengths and arbitrary field strength
}

\author{
O. A. Anderson, LBNL, Berkeley, CA 94720, USA \\ L. L. LoDestro, LLNL, Livermore, CA 94551, USA
}

(Dated: April 29, 2009)

\begin{abstract}
In 1958, Courant and Snyder analyzed the problem of alternating-gradient beam transport and treated a model without focusing gaps or space charge. Recently we revisited their work and found the exact solution for matched-beam envelopes in a linear quadrupole lattice [O.A. Anderson and L.L. LoDestro, Phys. Rev. ST Accel. Beams, 2009]. We extend that work here to include the effect of gaps. We derive the exact envelopes and show results for various field strengths, occupancies $\eta$, and gap-length ratios. We find the peak envelope excursion. It has a broad minimum as a function of the phase advance $\sigma$ (typically around $34^{\circ}$ ) over which it varies less than $\pm 1 \%$. The phase-advance numbers also change little over the full range of gap ratios. However, the required field strengths vary appreciably. In the second stable band, the higher field strength necessitated by the lower occupancy accentuates the remarkable compression effect predicted for the FD (gapless) model
\end{abstract}

\section{INTRODUCTION}

In their classic paper, Courant and Snyder [1] studied the envelope dynamics of a circular machine with negligible space charge, piecewise constant focusing, and no drift spaces (focusing gaps); they used an expansion in focusing strength to obtain an approximate solution for the envelope. The same case, but for a straight machine, was recently analyzed and an exact solution was obtained [2], [3]. In the present paper we extend that recent analysis to include focusing gaps, still assuming negligible space charge. Of course, particular cases with gaps have long been studied via computer simulations; an especially complete study (including space charge) was published by Lund and Bukh [4]. As with the simulations of Ref. [4], our analysis is not restricted to FODO; the general case of unequal drift lengths is analyzed. The motivations for finding the exact solution are: (1) ease of performing parametric studies such as those in Secs. VI and VIII; (2) to facilitate study of envelope functions in the higher bands [Sec. VII], where approximation methods fail and simulations become difficult. In particular, we are interested in the effect of drift spaces and asymmmetry on the remarkable second-band beam compression effect [Sec. IX] previously reported for the FD case [3]. The parametric studies, beam envelopes, and compression results are all summarized in Sec. ??.

Instead of solving the envelope equations directly, as we did in Ref. [3], we use here the linear single-particle equations and the phase-amplitude method to get the exact envelope functions and phase advances. To indicate briefly that, in our model, the periodic lattice of quadrupole doublets has piecewise-constant focusing but may have unequal gap lengths, we introduce the abbreviation $F_{O D O}$.

\section{FoDO FOCUSING MODEL}

We assume a focusing function $\kappa(z)$ that is periodic over a lattice with period $2 L$, so that $\kappa(z+2 L)=\kappa(z)$. We take $\kappa(z)$ to be piecewise constant with value $+\kappa_{\max }$ in the focus and $-\kappa_{\max }$ in the defocus sections, which have equal length. For convenience throughout, we define

$$
k \equiv \sqrt{\kappa_{\max }} .
$$

Our model is then described for the $x z$-plane by Eqs. (2) and Fig. 1:

$$
\kappa(z) \equiv \begin{cases}+k^{2}, & 0<z<\eta L \\ 0, & \eta L<z<\eta L+d_{1} \\ -k^{2}, & \eta L+d_{1}<z<2 \eta L+d_{1} \\ 0, & 2 L-d_{2}<z<2 L .\end{cases}
$$

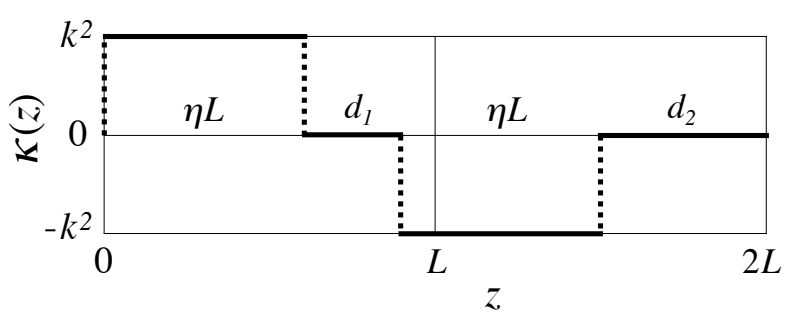

FIG. 1: Focusing model for one cell of a periodic FoDO lattice, which starts at $z=0$. The quadrupoles have equal lengths $\eta L$; the gap lengths are $d_{1}$ and $d_{2}$. The $x z$-plane, shown here, starts with $\kappa>0$ (focus). The $y z$-plane field map is the same except inverted; i.e., defocus comes first.

Since the FoDO lattice cell (Fig. 1) has equal focus and defocus lengths, the fields have antisymmetry about each gap center. For a matched beam, this yields a relationship between the envelopes $a(z)$ and $b(z)$ in the $x z$ and $y z$ planes, respectively. One finds that

$$
b(z)=a\left(2 z_{c}-z\right)
$$

where $z_{c}$ is the center of any gap. Therefore, we only need to analyze $a(z)$ in what follows. 


\section{DEFINITIONS}

\section{A. Gap asymmetry parameter $\mu$}

The average gap length $d$ is

$$
d \equiv \frac{d_{2}+d_{1}}{2}=(1-\eta) L .
$$

We define the gap asymmetry parameter

$$
\mu \equiv \frac{d_{2}-d_{1}}{2 d}
$$

$$
d_{1}=d(1-\mu), \quad d_{2}=d(1+\mu) .
$$

\section{B. The FoDO strength parameter $\varphi$}

The FoDO strength parameter, used throughout this paper, is defined by

$$
\varphi \equiv \eta k L \text {. }
$$

We introduce the following quantities that depend on the FoDO strength parameter:

$$
\begin{array}{llrl}
s n & \equiv \sin \varphi, & c s & \equiv \cos \varphi, \\
s h & \equiv \sinh \varphi, & c h \equiv \cosh \varphi
\end{array}
$$

and the normalized gap lengths

$$
\begin{aligned}
\nu & \equiv k d=k(1-\eta) L=\frac{1-\eta}{\eta} \varphi \\
\nu_{1} & \equiv k d_{1}=\nu(1-\mu) \\
\nu_{2} & \equiv k d_{2}=\nu(1+\mu) .
\end{aligned}
$$

Note that in the limit $\eta \rightarrow 1, \nu$ vanishes and $s n, c s, s h$, and $c h$ become identical with the functions defined in Ref. [3].

\section{ENVELOPE EQUATION}

For a beam with the KV distribution, emittance $\in$, negligible space charge, and arbitrary periodic focus function $f(z)$, the $x z$-plane envelope function $a(z)$ is determined by [5]:

$$
a(z)^{\prime \prime}+f(z) a-\frac{\epsilon^{2}}{a^{3}}=0
$$

along with initial or periodic conditions for $x$ and $y$. We assume $\epsilon_{x}=\epsilon_{y}=\epsilon$. (We also note that in the absence of space charge, the KV distribution can be replaced by a class of physically realistic distributions.)

For a matched beam without space charge, it is unnecessary to solve the nonlinear equation (12) directly. Instead, we begin with the linear single-particle equation

$$
x^{\prime \prime}(z)+f(z) x(z)=0
$$

and use the phase-amplitude method (see appendices) to find the envelopes.

\section{TRANSFER MATRICES FOR FoDO CASE}

The appendices give a short derivation of the envelope solution for the general case, Eq. (B4). We repeat the result here for convenience:

$$
\frac{1}{\epsilon} a^{2}(z)=\frac{M_{12}(z)}{\mathrm{P} \sqrt{1-\left(\frac{1}{2} \operatorname{Tr} \mathbf{M}\right)^{2}}} .
$$

(The sign function $\mathrm{P}$, defined by Eq. (B5), provides the correct sign for the radical for any phase advance [3].)

The matrix $\mathbf{M}$ is obtained by multiplying the transfer matrices for the segments of a lattice cell. In the case of a FoDO cell, these segments - taken in the order of Fig. 1-have transfer matrices [1], [6]

$$
\begin{aligned}
& \mathbf{M}_{F}=\left(\begin{array}{cc}
c s & \frac{1}{k} s n \\
-k s n & c s
\end{array}\right) \text {, } \\
& \mathbf{M}_{O_{1}}=\left(\begin{array}{cc}
1 & d_{1} \\
0 & 1
\end{array}\right), \\
& \mathbf{M}_{D}=\left(\begin{array}{cc}
c h & \frac{1}{k} s h \\
k s h & c h
\end{array}\right) \text {, } \\
& \mathbf{M}_{O_{2}}=\left(\begin{array}{cc}
1 & d_{2} \\
0 & 1
\end{array}\right) .
\end{aligned}
$$

The matrix for the entire cell, starting at $z=0$ in Fig. 1, is

$$
\mathbf{M}(0)=M(2 L)=\mathbf{M}_{O_{2}} \mathbf{M}_{D} \mathbf{M}_{O_{1}} \mathbf{M}_{F} .
$$

The ranges of $z$ for the four individual segments are indicated in Fig. 1, namely, $\eta L, d_{1}, \eta L$, and $d_{2}$. If $z \neq 0$ but, for example, $z$ lies within the first segment, then $\eta L$ splits into ranges $z$ and $\eta L-z$. There are then five component matrices, as in Sec. VII A.

\section{STABILITY AND PHASE ADVANCE}

A single-particle orbit is stable if $|\operatorname{Tr} \mathbf{M}|<2$ (see Eq. (B2)). The trace is independent of $z$ [4]; it is convenient to calculate it at $z=0$, using $\mathbf{M}(0)$ from Eq. (19).

First, we introduce a matrix that will be used again in Sec. VII A:

$$
\mathbf{M}_{\mathrm{III}} \equiv \mathbf{M}_{O_{2}} \mathbf{M}_{D} \mathbf{M}_{O_{1}}=\left(\begin{array}{cc}
A_{1} & \frac{2 B+s h}{k} \\
k s h & A_{2}
\end{array}\right) \text {, }
$$

with $A_{1}$ and $A_{2}$ defined by

$$
A_{1} \equiv c h+\nu_{2} s h, \quad A_{2} \equiv c h+\nu_{1} s h,
$$

and $B$ by

$$
B \equiv \nu \operatorname{ch}+\frac{1-\mu^{2}}{2} \nu^{2} s h
$$


Then

$$
\mathbf{M}(0)=\left(\begin{array}{cc}
A_{1} & \frac{2 B+s h}{k} \\
k s h & A_{2}
\end{array}\right)\left(\begin{array}{cc}
c s & \frac{1}{k} s n \\
-k s n & c s
\end{array}\right),
$$

It is unnecessary to write all the components of the full matrix in order to find the phase advance since, according to Eq. (B2), only $\mathbf{M}_{11}$ and $\mathbf{M}_{22}$ are needed. We find

$$
\cos \sigma=\frac{\mathbf{M}_{11}+\mathbf{M}_{22}}{2}=(c h+\nu s h) c s-B s n,
$$

which agrees with the result given by Lund and Bukh [4]. $\sigma$ is the phase advance for a whole period, defined by Eq. (A9). The envelope solution will be stable for all values of $\varphi$ for which the right-hand side of Eq. (23) lies within the range $[-1,1]$. Such regions of $\varphi$ are referred to as stable bands or pass bands. Reference [3] shows how these bands are related to the branches of $\cos \sigma$.

Using appropriate branches of Eq. (23), the phase advance is plotted as a function of $\sqrt{\kappa} L / \pi$ in Fig. 2 for various cases of occupancy $\eta$ and asymmetry $\mu$. The figure shows that introducing drift spaces requires a moderate increase in focus strength in the first band and a large increase in the second. (See the quantitative discussion of Figs. 3 and 4.)

\section{EXACT ENVELOPE FOR STABLE BANDS}

\section{A. Focus segment}

Now we are ready to use Eq. (14) to find the envelope in the $x z$ plane. For an arbitrary point $z$ in the first (focus) segment, the full-period transfer matrix is obtained from $\mathbf{M}_{\text {III }}$ after pre- and post-multiplying by the two subunits of $\mathbf{M}_{F}$ referred to in Sec. V. Using Eq. (20), we have

$$
\begin{aligned}
& \mathbf{M}^{\mathrm{f}}(z)=\left(\begin{array}{cc}
\cos k z & \frac{1}{k} \sin k z \\
-k \sin k z & \cos k z
\end{array}\right)\left(\begin{array}{cc}
A_{1} & \frac{2 B+s h}{k} \\
k \operatorname{sh} & A_{2}
\end{array}\right) \times \\
&\left(\begin{array}{cc}
\cos k(\eta L-z) & \frac{1}{k} \sin k(\eta L-z) \\
-k \sin k(\eta L-z) & \cos k(\eta L-z)
\end{array}\right) .
\end{aligned}
$$

The superscript $\mathrm{f}$ means that $z$ is restricted here to the focusing segment of the quadrupole cell. The matrix multiplications are a bit tedious, but to find $a(z)$ we only need the element $\mathbf{M}_{12}^{\mathrm{f}}$. It is:

$$
\begin{aligned}
2 k \mathbf{M}_{12}^{\mathrm{f}}=\left(A_{1}\right. & \left.+A_{2}\right) s n+\left(A_{1}-A_{2}\right) \sin [k(\eta L-2 z)] \\
& +2 B c s+(2 B+2 s h) \cos [k(\eta L-2 z)] .
\end{aligned}
$$

Finally, we define $F(\varphi, z) \equiv k \mathbf{M}_{12}^{\mathrm{f}}$ and use Eq. (7) to get

$$
\begin{array}{r}
F(\varphi, z)=(c h+\nu s h) s n+\mu \nu \operatorname{sh} \sin [\varphi(1-2 z / \eta L)] \\
+B c s+(B+s h) \cos [\varphi(1-2 z / \eta L)] .
\end{array}
$$
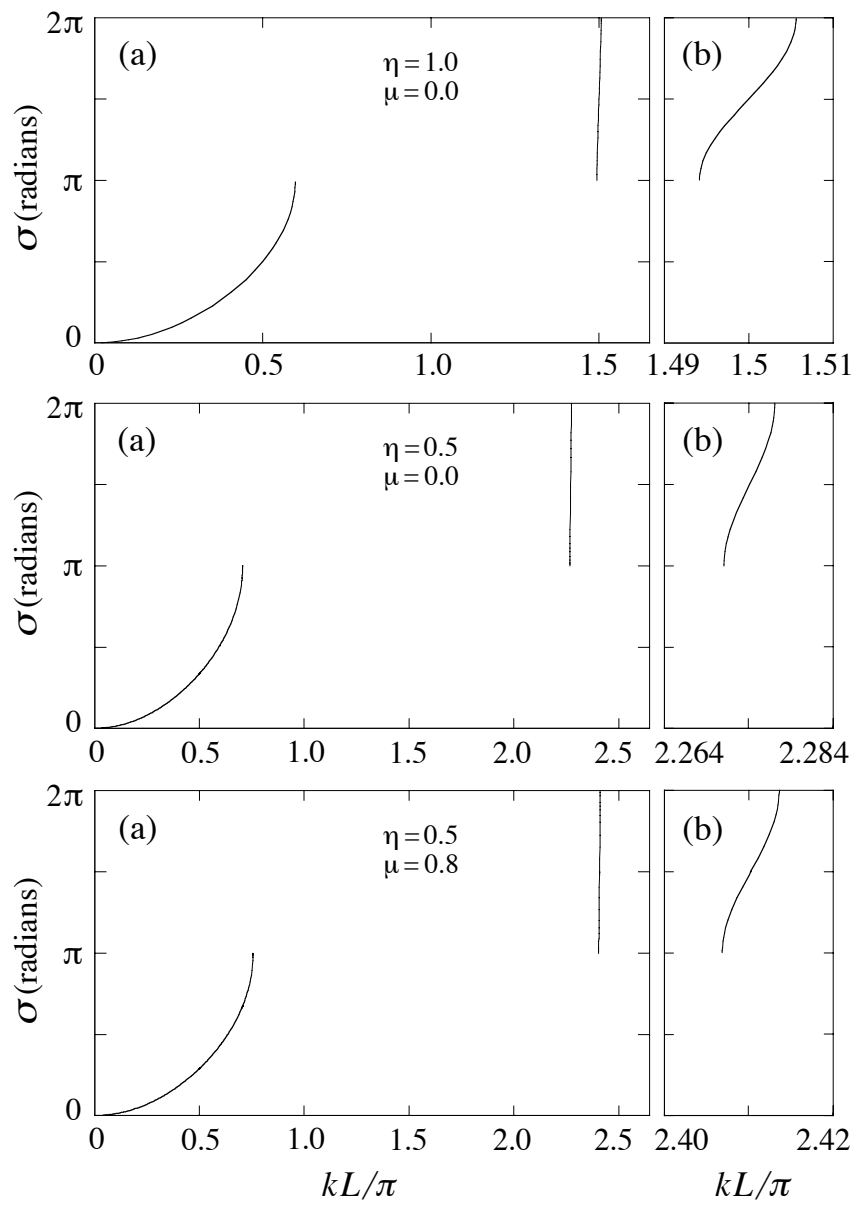

FIG. 2: (a) Phase advance from Eq. (23) for the first two stable bands. (b) The second band again with the $k L$ axis magnified. Occupancies are $\eta=1.0$ for the top figure and $\eta=0.5$ for the others. The bottom figure has gap asymmetry $\mu=0.8$. The figure shows that introducing drift spaces has a particularly large effect on the second pass band, requiring large increases in field strength and tighter regulation (see text).

Using Eq. (B4), the exact result for the focus-segment envelope is, then,

$$
a^{2}(\varphi, z)=\in \eta L \frac{F(\varphi, z)}{\mathrm{P} \varphi \sqrt{1-\left(\frac{1}{2} \operatorname{Tr} \mathbf{M}\right)^{2}}}
$$

with $\operatorname{Tr} \mathbf{M}(\varphi)$ from Eq. (23).

Note that full occupancy (i.e., $\nu=0$ ) implies that $\mu=0$ and $B=0$, so that Eq. (27) reduces to the previous result for FD focusing [3], which was derived by a different method.

\section{B. Drift and defocus segments}

We present the matched envelope functions for all four segments in a lattice cell graphically here. In the figures, 
$a(z)$ was obtained from the exact results, Eqs. (27), (C4), (C5), and (C6), while $b(z)$ was obtained from Eq. (3).

Figures 3 and 4 plot the normalized envelope functions $a_{\text {norm }} \equiv a(z) / \sqrt{\epsilon L}$ and $b_{\text {norm }} \equiv b(z) / \sqrt{\epsilon L}$ for various combinations of occupancy and gap asymmetry. The origin in these figures has been shifted from that in Fig. 1. It is placed at the center of the second drift space in order to display the matched-beam symmetry described in Sec. II. In Fig. 3, the second and third cases use the same parameters used by Lund, Chilton, and Lee [7] except that here $Q=0$. That is, we use the same occupancies, gap asymmetries, and (undepressed) phase advances $\left(80^{\circ}\right)$. Our results appear to be very similar to theirs.

It is remarkable that the peak envelopes in Fig. 3 are
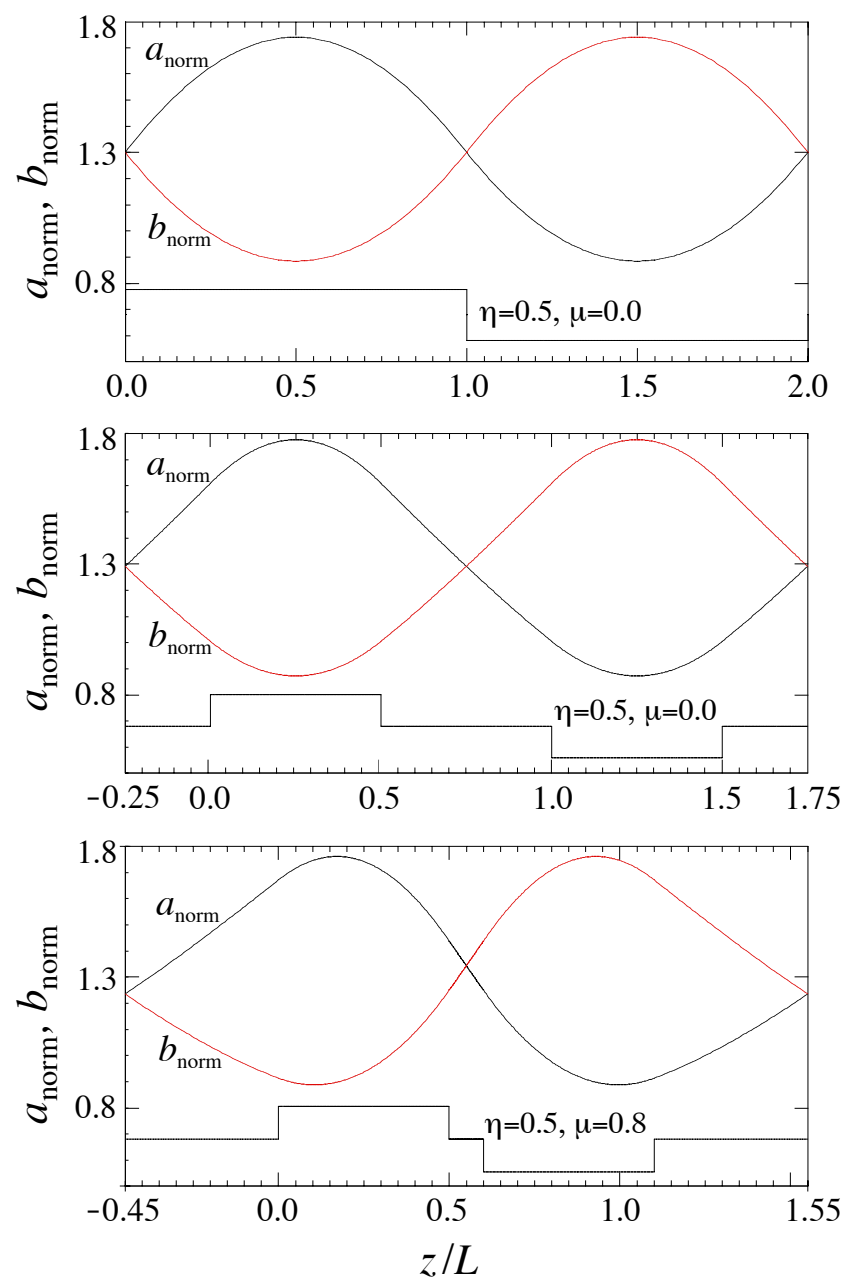

FIG. 3: Exact normalized envelope functions from Eqs. (27), (C4), (C5), and (C6). The normalized radii are defined by $a_{\text {norm }} \equiv a(z) / \sqrt{\in L}$ and similarly for $b_{\text {norm }}$. Lattice parameters are as in Fig. 2. Field strengths for all figures are adjusted to give $\sigma$ very close to $80^{\circ}$, approximately the midpoint of each band. The required focus strengths are $k L=0.47642 \pi$, $0.56546 \pi$, and $0.60565 \pi$, respectively. The field strengths $\kappa=k^{2}$ thus change considerably while the peak envelopes are kept nearly constant (see text).
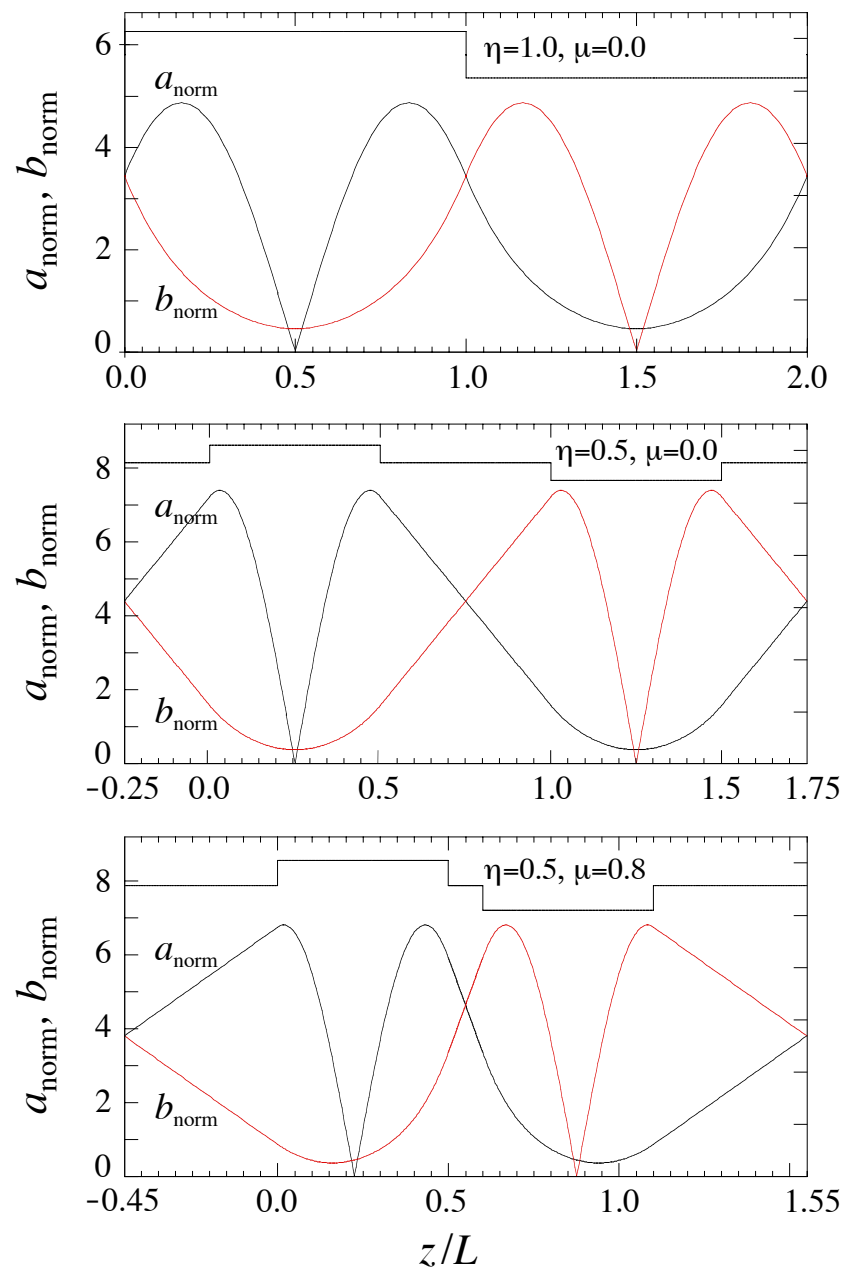

FIG. 4: Same as Fig. 3 [again using exact equations (27), (C4), (C5), and (C6)] but with focus parameter $k L$ increased to the middle of the second pass band shown in Fig. 2. Values are $k L=1.5 \pi, 2.27411 \pi$, and $2.41027 \pi$, respectively, which give phase advances equal to or very near to $\sigma=270^{\circ}$. The peak radii here, unlike those in the first band, increase considerably when the drift spaces are introduced (see text).

nearly identical $(1.740,1.776$, and 1.761). However, the lower occupancy in the second figure requires that, in order to maintain the phase advance of $80^{\circ}$, the field strength $\kappa L^{2}=(k L)^{2}$ must be increased over the value 0.22698 by the factor 1.409 . This factor becomes 1.616 for the third figure.

Figure 4 shows a different situation. In the second band, the drift spaces lead to significant increases in peak radius which vary considerably with the lattice parameters. The radii are $4.860,7.386$, and 6.742 , respectively. The field strength $\kappa L^{2}=2.25$ required for the top figure must be increased by the factor 2.2985 when the occupancy is reduced to 0.5 and by the factor 2.5820 with the addition of asymmetry parameter $\mu=0.8$. 


\section{PEAK VALUE OF THE ENVELOPE}

The peak envelope values (Fig. 5) always occur in the focus segment. In the higher stable bands, minimum values occur there as well. Defining $n$ as the pass-band number, with $n=1$ for the first band, there are $2 n-1$ extrema in the focus segment.
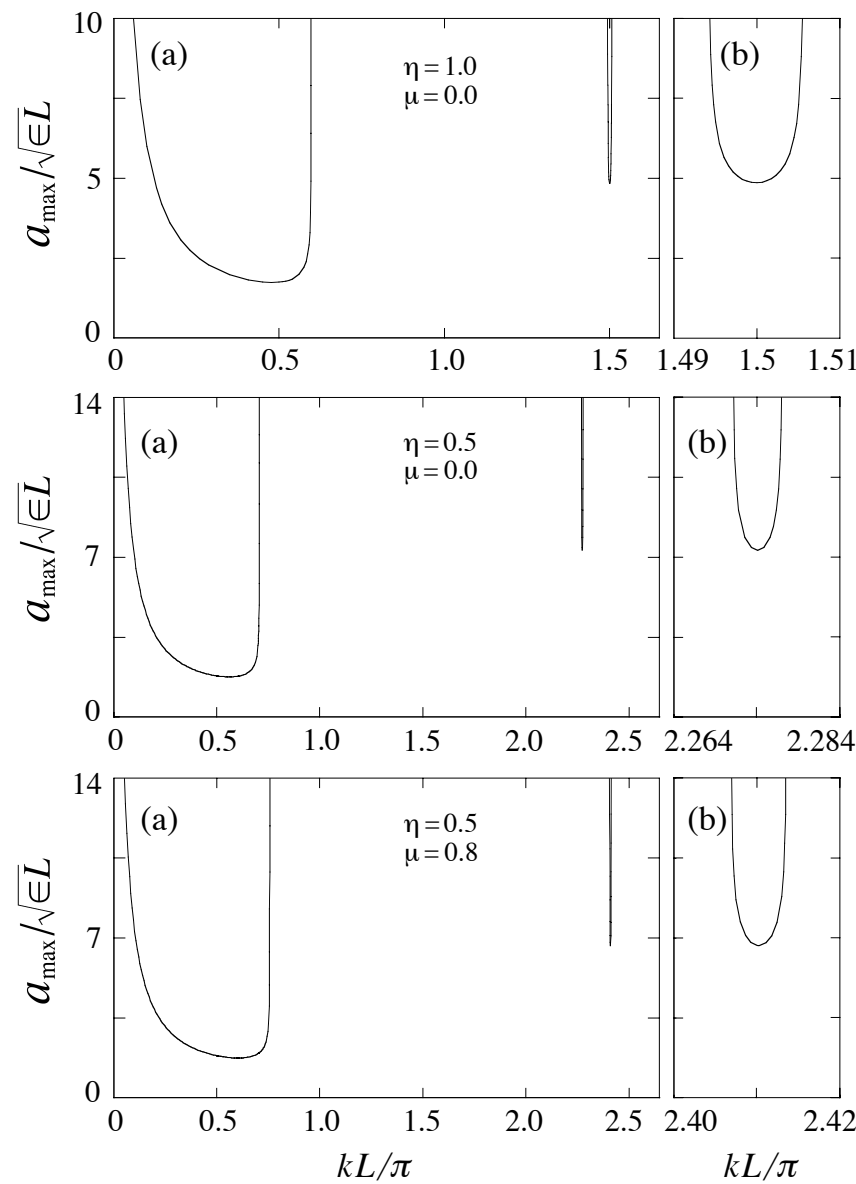

FIG. 5: Exact peak envelope functions from Eq. (32) as functions of $k L$. Same lattice parameters (occupancy and asymmetry) as in Figs. 2, 3, and 4. (a) First two stable bands. (b) Second band magnified. Note the vertical scale change for the last two figures.

Defining the midpoint $\varphi_{n}$ of the $n_{\mathrm{th}}$ band as the point where $\operatorname{Tr} \mathbf{M}\left(\varphi_{n}\right)=0$, we determine the locations $z_{m}$ of the envelope extrema by differentiating Eq. (26) with respect to $z$ and setting the result equal to zero. We find

$$
\begin{aligned}
& \varphi_{n}\left(1-2 z_{m} / \eta L\right)=\tan ^{-1} \frac{\mu \nu s h}{B+s h} \\
& \pm 0, \pm \pi, \pm 2 \pi, \pm \cdots, \pm(n-1) \pi
\end{aligned}
$$

with the principal value used for $\tan ^{-1}$. The maxima and minima occur where $z=z_{m}$. Next, we define

$$
\zeta_{n m} \equiv \varphi_{n}\left(1-2 z_{m} / \eta L\right)
$$

where $\zeta_{n m}$ is one of the angles allowed in Eq. (28).
In the first pass band, there is a single maximum, in the second, there is a minimum between two maxima, and so forth. The first extremum is always a maximum, so that

$$
\zeta_{n 1}=\tan ^{-1} \frac{\mu \nu s h}{B+s h}+(n-1) \pi
$$

always represents the maximum excursion of the envelope, and

$$
\begin{gathered}
F_{\max }(\varphi)=(c h+\nu s h) s n+\mu \nu \operatorname{sh} \sin \zeta_{n 1} \\
+B c s+(B+s h) \cos \zeta_{n 1}, \\
a_{\max }^{2}(\varphi)=\in \eta L \frac{F_{\max }(\varphi)}{\mathrm{P} \varphi \sqrt{1-\left(\frac{1}{2} \operatorname{Tr} \mathbf{M}\right)^{2}}} .
\end{gathered}
$$

These results are plotted in Fig. 5, showing the first two pass bands as in Fig. 2. The lattice parameters are the same as in the previous figures, and the discussion of those figures applies here.

\section{SECOND-BAND BEAM COMPRESSION}

Beam compression occurs in even bands due to envelope minima at or near the same $z$ in both the $x z$ and $y z$ planes. Ref. [3] shows that the effect becomes extreme near the outer band edge (but it notes that caveats apply). The effect is even larger when there are drift spaces because the focus strength must be increased. For $\eta=0.5, \mu=0.0$, and $\sigma=356.75$, the area compression ratio is $1.17 \times 10^{6}$. However, if the asymmetry parameter $\mu$ is finite, the $x z$ and $y z$ compression points become separated and, for $\mu=0.8$ (Fig. 6), the area compression ratio is only $7 \times 10^{4}$.

\section{Acknowledgments}

We thank Steve Lund and Ed Lee for suggesting the transfer matrix method of solution. This work was supported in part by the Director, Office of Science, Office of Fusion Energy Sciences, of the U.S. Department of Energy under Contract No. DE-AC02-05CH11231.

\section{APPENDIX A: PHASE-AMPLITUDE METHOD}

In their 1958 paper [1], Courant and Snyder described the phase-amplitude method of solution for equations having the form of Eq. (13) or (A1) and applied it to the alternating-gradient problem. They introduced and utilized a number of powerful concepts (beta function, Courant-Snyder invariant) which are not needed in our present work. This appendix serves to make our paper fairly self-contained by giving a relatively simple derivation of the solution, Eq. (14), that does not utilize those advanced concepts. 

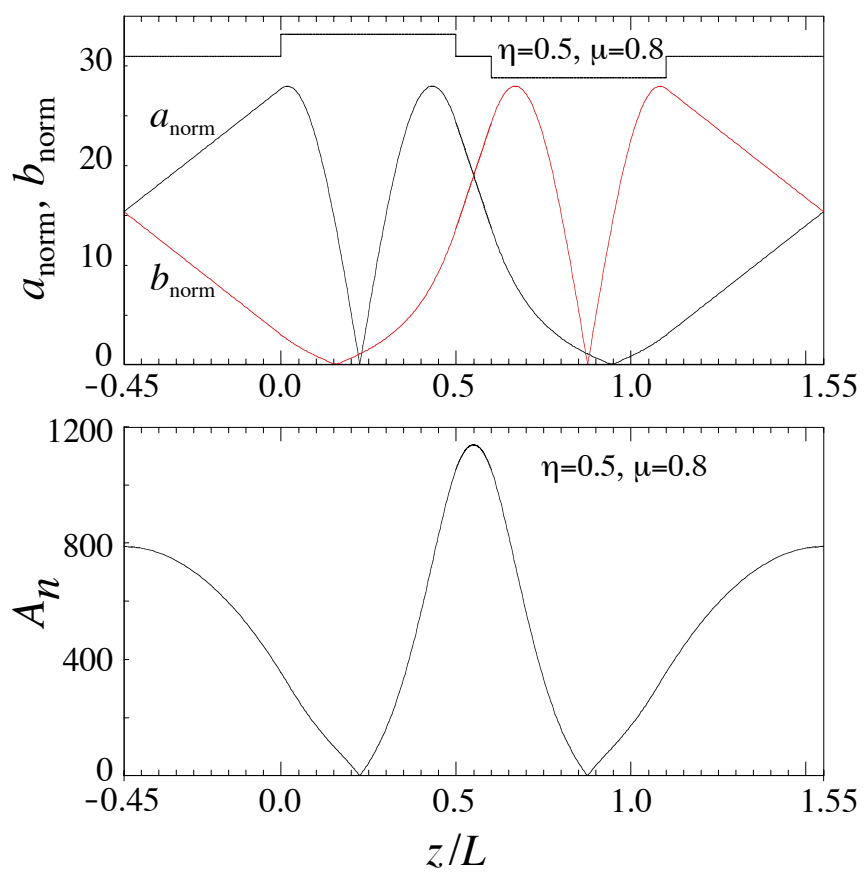

FIG. 6: Normalized envelope functions $a(z), b(z)$, and $A_{n}(z) \equiv \pi a b$ near outer edge of band 2: $k L=2.41361, \sigma=$ $356.6^{\circ}$. The beam compression $\left(7 \times 10^{4}\right)$ is much smaller than in Ref. [3] because the gaps have unequal length — see text.

Without space charge, the transverse position $x(z)$ of a single particle obeys the linear equation

$$
x^{\prime \prime}(z)+f(z) x(z)=0,
$$

One can verify by substitution that

$$
x=\frac{a(z)}{\sqrt{\epsilon}}\left[C_{1} \cos \psi+C_{2} \sin \psi\right]
$$

is the general solution of Eq. (A1), provided that $a(z)$ satisfies Eq. (12) and that

$$
\psi^{\prime}=\frac{\epsilon}{a^{2}} .
$$

The quantities $\psi(z)$ and $a(z)$ are known as the phase and amplitude. If we differentiate Eq. (A2) and use Eq. (A3), we get

$$
\sqrt{\epsilon} x^{\prime}=\left(C_{1} a^{\prime}+C_{2} \in / a\right) \cos \psi+\left(C_{2} a^{\prime}-C_{1} \in / a\right) \sin \psi
$$

Set $\psi\left(z_{0}\right)=0$. Then

$$
\begin{aligned}
& C_{1}=\sqrt{\epsilon} x_{0} / a_{0}, \\
& C_{2}=\left(a_{0} x_{0}^{\prime}-a_{0}^{\prime} x_{0}\right) / \sqrt{\epsilon} .
\end{aligned}
$$

Inserting these into Eq. (A2) gives

$$
\begin{aligned}
x(z) & =g_{11}\left(z, z_{0}\right) x_{0}+g_{12}\left(z, z_{0}\right) x_{0}^{\prime} \\
x^{\prime}(z) & =g_{21}\left(z, z_{0}\right) x_{0}+g_{22}\left(z, z_{0}\right) x_{0}^{\prime} .
\end{aligned}
$$

where the coefficients $g_{i j}$ are found from Eqs. (A2), (A4), (A5), and (A6). In App. B we will need the coefficient $g_{12}$, which is

$$
g_{12}\left(z, z_{0}\right)=\frac{1}{\epsilon} a_{0} a(z) \sin \psi
$$

At this point, we specify that the beam is matched, i.e., $a(z)$ is periodic with the same period, $2 L$, as the lattice. Then $a\left(z_{0}+2 L\right)=a\left(z_{0}\right)$. We define

$$
\psi\left(z_{0}+2 L\right) \equiv \sigma
$$

the phase advance for a whole period, and observe from Eq. (A3) that, for the matched beam, $\sigma$ is independent of the choice of $z_{0}$. Then, writing Eqs. (A7) in matrix form for the case $z=z_{0}+2 L$, we have

$$
\left(\begin{array}{c}
x(z+2 L) \\
x^{\prime}(z+2 L)
\end{array}\right)=\left(\begin{array}{ll}
g_{11}(z+2 L, z) & \frac{1}{\epsilon} a^{2}(z) \sin \sigma \\
g_{21}(z+2 L, z) & g_{22}(z+2 L, z)
\end{array}\right)\left(\begin{array}{c}
x(z) \\
x^{\prime}(z)
\end{array}\right) .
$$

We have dropped the subscript on $z_{0}$ because the location of $z_{0}$ is arbitrary. Note that the matrix elements here are periodic but $x(z)$ is not. If one writes out $g_{11}$ and $g_{22}$, one sees that the trace has the value

$$
g_{11}+g_{22}=2 \cos \sigma,
$$

which is independent of $z$.

\section{APPENDIX B: MATCHED ENVELOPE SOLUTION}

Now we are ready to calculate the matched beam envelope for a machine with periodic focusing function $f(z)$. In general, there is a machine matrix $\mathbf{M}(z)$, obtainable by integrating Eq. (A1), that gives the phase-space change over a whole machine period (for the specific FoDO example, see Secs. VI and VII A):

$$
\left(\begin{array}{c}
x(z+2 L) \\
x^{\prime}(z+2 L)
\end{array}\right)=\left(\begin{array}{ll}
M_{11}(z) & M_{12}(z) \\
M_{21}(z) & M_{22}(z)
\end{array}\right)\left(\begin{array}{c}
x(z) \\
x^{\prime}(z)
\end{array}\right) .
$$

Comparing Eqs. (B1) and (A10) shows that

$$
2 \cos \sigma=M_{11}+M_{22}=\operatorname{Tr} \mathbf{M}
$$

and

$$
M_{12}=\frac{1}{\epsilon} a^{2}(z) \sin \sigma
$$

or

$$
\frac{1}{\epsilon} a^{2}(z)=\frac{M_{12}(z)}{\mathrm{P} \sqrt{1-\left(\frac{1}{2} \operatorname{Tr} \mathbf{M}\right)^{2}}} .
$$

The $\mathrm{P}$ function, defined by

$$
\mathrm{P}(\sigma) \equiv \operatorname{sign}(\sin \varphi),
$$

gives the correct branch of the radical and ensures that the right-hand side of Eq. (B4) is always positive. 


\section{APPENDIX C: EXACT ENVELOPES IN DRIFT AND DEFOCUS SEGMENTS}

In the main text, we gave details of the envelope calculation for the focus segment. Here, we briefly describe our method for the remaining segments and give the results.

The envelope calculation for any segment is simplified by moving the origin to the beginning of the segment. Thus, the matrices that will yield the envelopes for the three remaining segments are

$$
\begin{gathered}
\mathbf{M}^{0_{1}}(z)=\left(\begin{array}{ll}
1 & z \\
0 & 1
\end{array}\right) \mathbf{M}_{F} \mathbf{M}_{O_{2}} \mathbf{M}_{D}\left(\begin{array}{cc}
1 & d_{1}-z \\
0 & 1
\end{array}\right), \\
\mathbf{M}^{\mathrm{d}}(z)=\left(\begin{array}{cc}
\cosh k z & \frac{1}{k} \sinh k z \\
k \sinh k z & \cosh k z
\end{array}\right) \mathbf{M}_{O_{1}} \mathbf{M}_{F} \mathbf{M}_{O_{2}} \times \\
\\
\left(\begin{array}{cc}
\cosh k(\eta L-z) & \frac{1}{k} \sinh k(\eta L-z) \\
k \sinh k(\eta L-z) & \cosh k(\eta L-z)
\end{array}\right), \\
\mathbf{M}^{0_{2}}(z)=\left(\begin{array}{ll}
1 & z \\
0 & 1
\end{array}\right) \mathbf{M}_{D} \mathbf{M}_{O_{1}} \mathbf{M}_{F}\left(\begin{array}{cc}
1 & d_{2}-z \\
0 & 1
\end{array}\right) .
\end{gathered}
$$

Note that the cyclic order is maintained in all cases.

After carrying out the matrix multiplications, one obtains the envelopes from $\mathbf{M}^{0_{1}}{ }_{12}, \mathbf{M}_{12}^{\mathrm{d}}$, and $\mathbf{M}^{0_{2}}{ }_{12}$. For the first drift segment, the numerator in Eq. (B4), which gives the exact envelope, is

$$
\mathbf{M}^{0_{1}}{ }_{12}=G_{1}-2 H_{1} k z+J_{1} k^{2} z^{2},
$$

where

$$
\begin{aligned}
& G_{1} \equiv 2 \nu \operatorname{chcs}+\nu_{1} \operatorname{sh} s n+\operatorname{chsn}+(1+2 D) \operatorname{shcs} \\
& H_{1} \equiv(1+D) \text { shsn }+\nu \text { chsn }+\mu \nu \operatorname{sh} c s ; \\
& J_{1} \equiv \nu_{2} s h s n+\text { chsn }- \text { shcs. }
\end{aligned}
$$

The numerator for the defocus segment is

$$
\mathbf{M}_{12}^{\mathrm{d}}=E_{d} s h+B_{d} c h+\mu \nu \operatorname{sn} \sinh \zeta+\left(s n+B_{d}\right) \cosh \zeta,
$$

where

$$
\begin{aligned}
B_{d} & \equiv \nu c s-D s n \\
E_{d} & \equiv c s-\nu s n \\
\zeta & \equiv \varphi(1-2 z / \eta L) .
\end{aligned}
$$

For the second drift segment,

$$
\mathbf{M}^{0_{2}}{ }_{12}=G_{2}+2 H_{2} k z+J_{2} k^{2} z^{2},
$$

where

$$
\begin{aligned}
G_{2} & \equiv 2 \nu \operatorname{chcs}-\nu_{2} \operatorname{sh} n+(1-2 D) \text { chsn }+ \text { shcs } \\
H_{2} & \equiv(1-D) \text { shsn }-\mu \nu \operatorname{ch} n+\nu \text { shcs } \\
J_{2} & \equiv \nu_{1} \text { shsn }+ \text { chsn }- \text { shcs. }
\end{aligned}
$$

[1] E. D. Courant and H. S. Snyder, Ann. of Phys. 3, 1 (1958)

[2] O. A. Anderson and L. L. LoDestro, in Proc. 2007 Particle Accelerator Conference, Albuquerque, NM, IEEE (2007).

[3] O. A. Anderson and L. L. LoDestro, Phys. Rev. ST Accel. Beams 12 (2009).

[4] S. M. Lund and B. Bukh, Phys. Rev. ST Accel. Beams 7, 024801 (2004)
[5] I. M. Kapchinskij and V. V. Vladimirskij, in Conf. on High Energy Accel. and Instrum. (CERN Sci. Inf. Service, Geneva, 1959), p. 274.

[6] M. Reiser, Particle Accelerators 8, 167 (1978).

[7] S. M. Lund, S. H. Chilton, and E. P. Lee, Phys. Rev. ST Accel. Beams 9, 064201 (2006). 